\title{
Bartonellosis: Suggestive Case Reports in Adult and Pediatric Patients and Therapeutic Issues
}

\author{
Roberto Manfredi, Sergio Sabbatani
}

\author{
Department of Clinical and Experimental Medicine, Division of Infectious \\ Diseases, University of Bologna “Alma Mater Studiorum”, S. Orsola \\ Hospital, Bologna, Italy
}

\begin{abstract}
Cat-scratch disease warrants extensive investigation, from an epidemiological, a diagnostic, but especially a therapeutic point of view. Two suggestive episodes of Bartonella henselae-caused catscratch disease are reported, and discussed in the light of the most recent literature evidence. The first case occurred in a 60-year-old man, thus suggesting that it is important to maintain an elevated level of suspicion for this disease in adults as well. Both episodes were characterized by a very prolonged and complicated disease course (with the involvement of three lymph node sets in the first case), a need for lymph-node drainage, and apparently negligible activity of many antimicrobial courses, with a very slow local cure. While specific culture and molecular biology techniques proved negative (probably due to late availability of appropriate clinical specimens), indirect immunofluorescence antibody assay was positive since the first weeks of disease, and elevated levels were also fond many months after disease onset. When clinicians face patients with prominent swelling of lymph nodes draining from the upper limbs, cat-scratch disease may be suspected on the grounds of epidemiological and clinical features, with a limited systemic involvement contrasting with a prominent local disease. The significance of specific antibody temporal kinetics in the subacute disease course is still unknown. Although biomolecular assays are now available, the time elapsed from disease onset to clinical diagnosis usually hampers diagnosis, while the roles of surgical debridement and of the unpredictable activity of antimicrobial chemotherapy warrant careful investigation.

Key Words: Adults, antimicrobial chemotherapy, Bartonellosis, Bartonella henselae, case reports,
\end{abstract} children, clinical presentation, outcome, treatment.

The spectrum of human disease caused by Bartonella spp. has expanded rapidly over the past two decades, prompted by the association of cat-scratch disease, AIDS-related bacillary angiomatosis, and bacillary peliosis with the fastidious Gram-negative oxidase-negative bacillus initially identified as Rochalimaea henselae, and subsequently classified within the genus Bartonella, on the grounds of genomic sequence analysis [1-3]. Five major Bartonella species have been found to be pathogenic for humans worldwide ( $B$. bacilliformis, B. henselae, B. quintana, B. elizabethae, and recently, B. clarridgeiae), but several other Bartonella spp. have been found in animal reservoirs, especially rodents and moles [1-5]. Up to $40 \%$ of domestic cats may have an asymptomatic $B$. henselae infection accompanied by bacteremia, which can persist for more than a year; this represents the most obvious risk factor for cat-scratch disease.

Two case reports of cat-scratch disease with distinguishing features, characterized by adult onset in the first patient and a very long course accompanied by persistently positive serologic assays occurring in both episodes, are described and discussed.

Received on 10 June 2006; revised 17 November 2006.

Address for correspondence: Prof. Roberto Manfredi. Infectious Diseases, University of Bologna, S. Orsola Hospital, Via Massarenti 11, I-40138 Bologna, Italy. Telephone: +39-051-6363355. Fax: +39051-343500.E-mail: Roberto.manfredi@ unibo.it.

The Brazilian Journal of Infectious Diseases 2006;10(6):411-415. (C) 2006 by The Brazilian Journal of Infectious Diseases and Contexto Publishing. All rights reserved.

\section{Case Reports}

A 60-year-old otherwise healthy male, two weeks after a mild domestic cat scratch, suffered from diffuse arthromyalgia, moderate fever, malaise and fatigue; he was referred by his primary care physician after lack of response to a three-week treatment with co-amoxiclav, followed by ciprofloxacin. At the time of our evaluation, only a small scar was visible on the dorsal portion of the right hand, accompanied by surrounding red papules, with prominent involvement of right epitrochlear and supraclavicular lymph nodes, characterized by painful swelling, skin reddening, and a slowly-progressing tendency to abscess formation in the forearm. Notwithstanding three further weeks of antimicrobial treatment carried out with ceftriaxonem followed by levofloxacin, and the disappearance of fever and constitutional symptoms, lymph node involvement extended to multiple axillary sites, and the epitrochlear localization (diameter $4 \mathrm{~cm}$ ) needed drainage of abundant and frankly purulent material, which tested negative in all culture examinations. Despite repeated local medications, continued spontaneous drainage of purulent material was observed in the subsequent four weeks of follow-up, with formation of a granulating ulceration with indurated edges and extensive erythematous infiltration of subcutaneous tissue. Surgical debridement allowed histopathological examination of epitrochlear lymph node sections (Figure 1), but both culture and bio-molecular searches for microbes (including Bartonella spp.) remained repeatedly negative. The laboratory work did not disclose relevant abnormalities of 
Figure 1. Lymph node section from our first patient, showing a diffuse chronic granulomatous inflammation characterized by epithelioid and giant cells, and palisade hystiocytes surrounding sparse necrotizing foci, and a moderate concurrent inflammatory infiltrate containing lymphoid and macrophage cells. Absence of fungal, mycobacterial, and Leishmania pathogens was confirmed after appropriate staining. Hematoxylin-eosin stain, original magnification 200-400x.
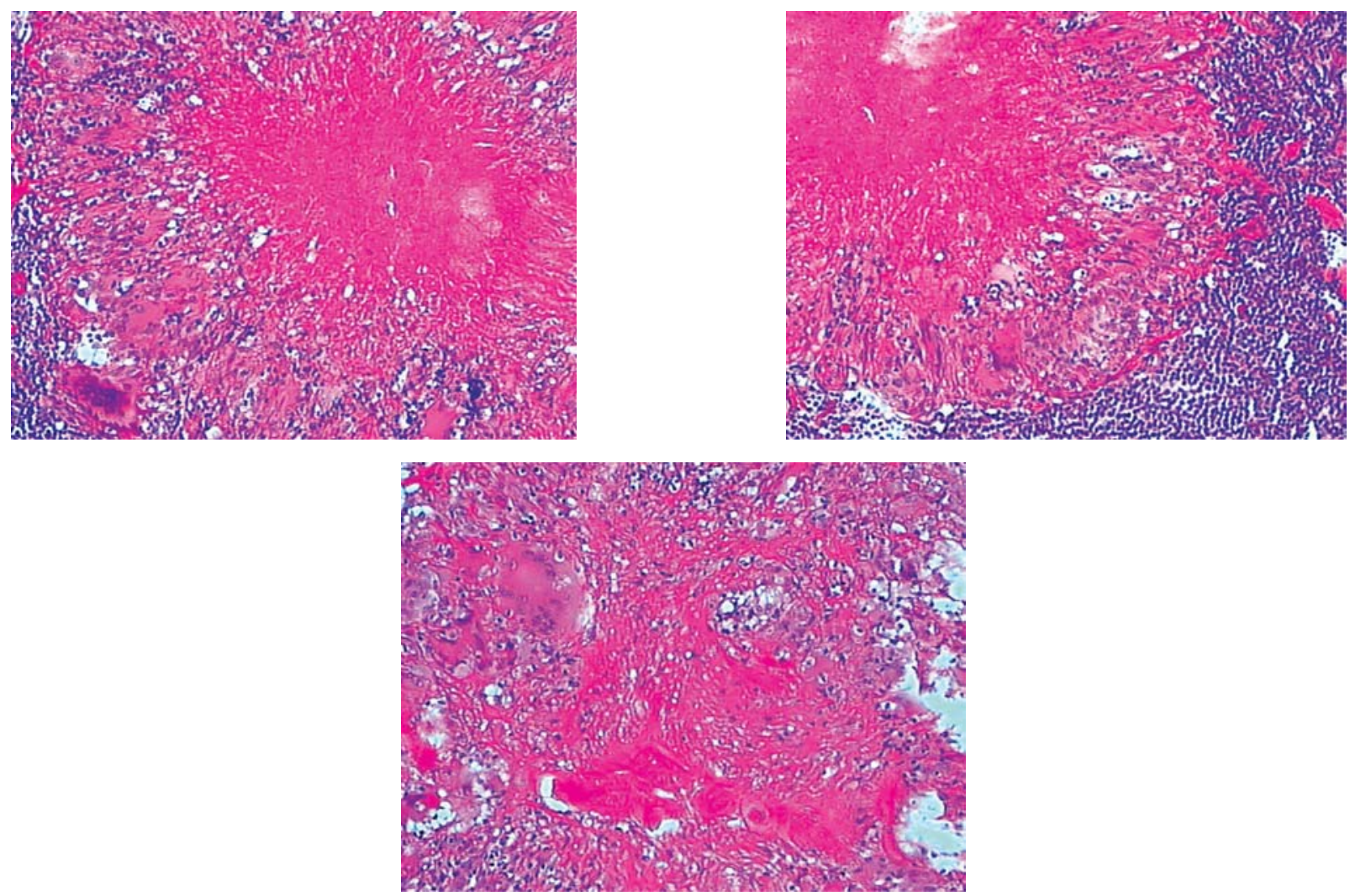

white blood cell counts, differential serum biochemistry profiles, or erythrocyte sedimentation rates (ESR). Serological testing for Toxoplasma gondii, Rickettsia spp., Borrelia burgdorferi, Leishmania spp., Brucella spp., syphilis, and HIV were negative, while elevated anti-Bartonella henselae antibodies were disclosed by an indirect immunofluorescence assay (IFA) at a titre of 1:256, followed by an increase up to 1:512 three weeks thereafter. After a further three-week treatment with associated tetracyclines and co-amoxiclav and continued local medications, the right forearm ulcer progressed to a very slow resolution in the following two months, while ultrasonographic evaluation confirmed multiple enlarged axillary lymph nodes with irregular morphology and a dishomogeneous echographic structure (maximum size $2.2 \mathrm{x}$ $1.2 \mathrm{~cm}$ ) and a persistent lymphangitis of the right arm. Repeated Bartonella serology testing showed a lower titre (1:256) only six months after the first examination. Interestingly, the two domestic cats in the household proved negative for Bartonella spp. infection after careful veterinary assessment (blood culture plus serology).

A nine-year-old boy came to our attention 10 days after a scratch by a domestic pet cat on his left forearm, owing to mild hyperpyrexia and increasing omolateral tender axillary lymph node swelling. At the time of first observation, four to five red papules and a painful lymphangitis were already present, while no other systemic signs and symptoms were appreciable. Laboratory examination showed a moderate leukocytosis with proportional neutrophilia (white blood cell count 12,600 cells/ $\mu \mathrm{L}$, and absolute neutrophils 52\%), increased ESR (26, first hour), with absence of alterations of the main biochemistry indexes and with a negative skin tuberculin assay. Serologic testing proved negative for acute Toxoplasma gondii, Cytomegalovirus, Borrelia burgdorferi, Leishmania spp., Brucella spp., and Francisella spp. infection, while antibodies against Bartonella henselae were found (1:128 after four weeks of disease). Despite multiple therapeutic attempts with amoxicillin, cefriaxone, azithromycin, cotrimoxazole, and coamoxiclav for an overall period of six weeks, and the achievement of local cure at the inoculation site and satellite lymphangitis, the axillary lymph node swelling progressively worsened, with multiple dishomogeneus nodes, characterized by hypodense round irregular lesions recognized on ultrasonography, progressive abscess formation with involvement of overhanging skin; spontaneous fistulization occurred after five weeks, while all other laboratory indexes returned to normal values, except for anti-Bartonella antibodies, which remained positive at the same titre (1:128) at nine months after diagnosis. Cultural examination of 
purulent discharge proved negative for all tested pathogens, and a very slow cure was attained after 2.5 months of continued local medications, with a minor local scar persisting at the left axil. Microscopic evaluation of clinical specimens of lymph node smears showed an aspecific granulomatous process, with a predominant macrophage-lymphoid infiltrate and sparse giant and epithelioid cells; there was absence of recognizable bacteria, mycobacteria, fungi, and protozoa, and a negative polymerase chain reaction for Bartonella spp. Reticulum cell hyperplasia and multiple necrotizing microabscesses were also recognized during the advanced-phase lymph node histological examination, carried out three months after disease onset. Clinically- and ultrasonography-appreciable axillary lymph nodes remained until nearly 12 months from disease onset.

\section{Discussion}

The most common clinical presentation of $B$. henselae infection is cat-scratch disease, a subacute, regional lymphadenitis, usually persisting for three weeks or more. This disorder, first thought to be caused by Afipia felis, has subsequently been attributed to $B$. henselae since 1992 , when isolation was made from healthy cats and reliable diagnostic testing was developed for humans [1,2,4-6]. Recently, another species of Bartonella (B. clarridgeiae) has also been associated with rare cases of cat-scratch disease $[2,3]$. Bartonella henselae antibody prevalence ranges from $84 \%$ to $100 \%$ in patients with cat-scratch disease; more sophisticated techniques allow culture from the involved lymph nodes, and detection of specific genomic sequences of $B$. henselae is possible with PCR techniques and other molecular biology assays [4,5].

From an epidemiological point of view, 87-99\% of patients with cat-scratch disease report some contact with cats (infection is caused by a cat flea, Ctenocephalides felis), although the exact mechanism of cat-to-human transmission remains in part unknown, since a scratch (or sometimes a bite) is documented in around half of the episodes; this disease usually involves children and adolescents, although it can occur at any age $[1,3,6]$. A study of 130 seropositive cases in Japan documented that $79 \%$ of episodes occurred under the age of $18[3,7,8]$, although adults can be involved, as documented by our case report. Extensive studies of $B$. henselae seroprevalence are still lacking; however, such an infection does not seem to occur frequently in the absence of suggestive signs and symptoms [9]. Moreover, in our first case the domestic cats tested negative for $B$. henselae infection in both culture and serology examination.

The incubation period of cat-scratch disease is estimated to be 7-15 days; some local non-pruritic papules or papulevesicles (often overlooked) may follow the inoculation lesion and precede the characteristic regional lymph node involvement. Usually the first or second set of lymph nodes draining the portal of entry site are affected (cervical, axillary, or epitrochlear localizations), but in rare cases more distant sites may also be involved (as in our first reported patient, who also had a supraclavicular involvement) [1-5,6-8].

The duration of lymph node enlargement is usually limited to one to two months, but persistence up to one year or more has been infrequently described [2-6]; our cases also had a very long course, despite multiple antimicrobial attempts and surgical drainage. The so-called "Parinaud oculoglandular syndrome" is characterized by a conjunctival site of entry, followed by preauricular lymphadenopathy $[3,7,10]$. Although cat-scratch disease is usually a self-limiting disorder within a few weeks or months, a locally severe disorder (as in our patients), or a disseminated illness may occur in a small percentage of cases. Granulomatous disease of the liver, spleen, mesenteric lymph nodes, heart (endocarditis) and also bone, with predominating general or focal signs and symptoms, has been reported [2-6,10,11]. Encephalopathy may occur as a complication in around $5 \%$ of patients, with sudden onset of a broad spectrum of neurological disturbances (seizures, altered behavior or consciousness, peripheral facial nerve paresis, myelo-radiculitis), two to three weeks after disease onset, but it usually has a favorable evolution $[2,4,6]$. Hematological manifestations of $B$. henselae disease may include hemolytic anemia, thrombocytopenia, and eosinophilia; rare cases of leukocytoclastic vasculitis, mediastinal masses, atypical pneumonia and pleural effusion, and other disease pictures mimicking collagen vascular diseases, have been reported in anecdotal cases [2-6,11]. As a consequence, the differential diagnosis of cat-scratch disease virtually includes all possible causes of lymphadenopathy: for instance, pyogenic and Toxoplasma gondii lymphadenitis, atypical mycobacterial infection, hematological and solid-organ malignancies, tularemia, and brucellosis [1-6]. In a study of 454 patients with unclear masses in the head and neck, 61 subjects (13.4\%) had confirmed cat-scratch disease [12]. Moreover, in a recent series, prolonged fever of unknown origin was the predominating sign in $2.8 \%$ of 130 cases; around $30 \%$ of these latter patients with a documented serological $B$. henselae infection lacked any lymphadenopathy [7]. From a pathogenetic point of view, the role of immune effector cells producing angiogenic cytokines upon stimulation with $B$. henselae has been recently claimed for immunocompromised hosts developing bacillary angiomatosis and peliosis, while the slow-progressing granulomatous response of otherwise healthy patients, typically found in cat-scratch disease, might be also triggered by an enhanced immune response to bacterial antigens $[4,5,13]$.

Cat-scratch disease may be strongly suspected on epidemiological and clinical grounds, but affordable serologic assays are available, such as commercial indirect immunofluorescence assay (IFA), hemagglutination, and 
more recently, enzyme-linked assays, which show good correlation with infection and disease; although comparative studies between the different available techniques are still lacking, timing of IgG and IgM response seems very variable, and cross-reactivity among the different Bartonella spp. organisms is expected [1$4,9,14,15]$. A long-term parallel serological and clinical follow-up of cat-scratch disease was recently published by Metzkor-Cotter and coworkers [15], using an enzymelinked immunoassay to monitor $B$. henselae antibody levels. Ninety-eight patients were followed for a median of 35.3 weeks: anti- $B$. henselae $\operatorname{IgM}$ antibodies were detected in $53 \%$ of subjects and remained positive up to three months after disease onset, while IgG titres decreased over time; $25 \%$ of patients remained seropositive for more than one year [15], which would explain the long-term positive IFA serology in our patients. Unfortunately, no association has been found between antibody titers or their kinetics and the evolution of clinical manifestations (typical or atypical), or with the overall illness duration or time to complete cure [15]. Very recently, a coupled immunofluorescence test applied to both serum and lymph node smears has significantly improved sensitivity and specificity of diagnosis of cat-scratch disease (up to $97.4 \%$, when at least one of these two tests was positive), and could be proposed for settings in which molecular biology assays are still not available [16]. In fact, although serology is the cornerstone for etiological diagnosis of cat-scratch disease, the sensitivity of the different serologic techniques can vary according to the antigen used, cut-off levels chosen, and the test procedures, so that results obtained in different series coming from different centers are not comparable $[9,14,15]$. Culturing, polymerase chain reaction, and other biomolecular studies are usually only available at reference centers $[4,12,14]$. Recovery from the blood requires prolonged incubation and use of highly-enriched media, or cultivation in endothelial cell lines, while $B$. henselae DNA can only be recovered during the first six weeks of disease, and late sample testing is usually negative. As a consequence, in common clinical practice the resort to biomolecular assays is often frustrating, since most patients came to the attention of a specialist some weeks after first disease appearance, and the incubation period is often overlooked. This situation may explain the lack of a positive culture or biomolecular assay in both our cases.

Routine hematological and biochemistry laboratory testing is normally not very useful: hepatic transaminases may be elevated in the rare cases of systemic involvement. Ultrasonography and/or CT examination give a better description of enlarged lymph nodes and may disclose the infrequent granulomatous involvement of visceral organs [13]. Diagnostic imaging may also give some help in the differential diagnosis of the most common localization of lymphadenopathy (that involving epitrochlear region); a recent magnetic resonance imaging (MRI) study has allowed the differential diagnosis of lymphadenopathy at this site, which was mainly caused by $B$. henselae regional infection alone [17].

Antimicrobial chemotherapy of cat-scratch disease is one of the most discussed and unresolved issues, including the need for, eventual selection, and duration of treatment. While some authors claim that no controlled evidence is available to determine benefits (such as reduction of severity and duration of disease), compared with conservative symptomatic care and follow-up, in immunocompetent, otherwise healthy pediatric and adult patients, other investigations tend to attribute a significant role of a broad spectrum of antibiotic treatments [2-6,18]. Currently, most Bartonella spp. isolates appear to be susceptible in vitro to a wide range of betalactams, as well as to rifampicin, erythromycin, and tetracyclines, while sensitivity to clindamycin, systemic quinolones, and cotrimoxazole seems more variable [2-5,19]. Unfortunately, a remarkable discordance between in vitro and in vivo activity of several molecules has been demonstrated. Controlled clinical data are very scarce, and the majority of studies report a poor response to penicillin derivatives and other compounds, despite apparent in vitro susceptibility $[6,19]$. A single placebo-controlled trial with azitromycin described some initial benefit, but contrasting long-term results [18]. Also in our cases, multiple antibiotic cycles carried out with different compounds did not seem to significantly modify the disease course in terms of time and mode of cure. Therefore, the role and mode of antimicrobial therapy in the setting of cat-scratch disease warrants more investigation. If treatment seems clinically indicated, new macrolides, cotrimoxazole, rifampicin, fluoroquinonoles, and gentamicin might be the firstchoice agents $[1,3,18,19]$. Corticosteroid adjuncts have been recently suggested for patients with a long-lasting course [20], whenever an exaggerated immune response has been found $[4,5,13]$, but no controlled data are available yet.

Suppurative lymph nodes that become tense, tender, and painful should be drained, but incision of non-suppurative lesions should be avoided, because chronic draining fistulae or very low tendency to cure may result $[2,3,6,8]$. When considering prevention, since interhuman transmission of Bartonella spp. disease is not documented, isolation measures are not necessary. Concomitant disease may occur in siblings, although very infrequently [21]. Preventive measures would require avoidance of close contacts with cats and cat fleas, with especially improved awareness of the risk of cat (especially kitten) scratches, at any age.

\section{References}

1. Maguiña C., Gotuzzo B. Bartonellosis: new and old. Infect Dis Clin North Am 2000; 14:1-22.

2. Windsor J.J. Cat-scratch disease: epidemiology, aetiology and treatment. Br J Biomed 2001;58:101-10.

3. Mansueto P, Di Lorenzo G, Rizzo M, Mazzola G, Affronti M, Battista Rini G, et al. Bartonellosi. Recenti Prog Med 2003;94:177-85. 
4. Jacomo V., Kelly P.J., Raoult D. Natural history of Bartonella infections (an exception to Koch's postulate). Clin Diagn Lab Immunol 2002;9:8-18.

5. Greub G., Raoult D. Bartonella: new explanations for old disease. J Med Microbiol 2002;51:915-23.

6. Carithers H.A. Cat scratch disease: an overview based on a study of 1,200 patients. Am J Dis Child 1985;139:1124-33.

7. Murakami K., Tsukahara M., Tsuneoka H., et al. Cat scratch disease: analysis of 130 seroposive cases. J Infect Chemother 2002;8:349-52.

8. Di Lorenzo G., Rizzo M., Mansueto P., et al. Tre casi di bartonellosi. Ann Ital Med Int 2002; 17:256-60.

9. Sander A., Berner R., Ruess M. Serodiagnosis of cat scratch disease: response to Bartonella henselae in children and a review of diagnostic methods. Eur J Clin Microbiol Infect Dis 2001;20:392-401.

10. Mirakhur B., Shah S.S., Ratner A.J., et al. Cat scratch disease presenting as orbital abscess and osteomyelitis. J Clin Microbiol 2003;41:3991-3.

11. Rolain J.M., Chanet V., Laurichesse H., et al. Cat scratch disease with lymphadenitis, vertebral osteomyelitis, and spleen abscesses. Ann N Y Acad Sci 2003;990:397-403.

12. Ridder G.J., Boedeker C.C., Technau-Ihling K., et al. Role of cat-scratch disease in lymphadenopathy in the head and neck. Clin Infect Dis 2002;35:643-9.

13. Resto-Ruiz S., Burgess A., Anderson B.E. The role of host immune response in pathogenesis of Bartonella henselae. DNA Cell Biol 2003;22:431-40.
14. Agan B.K., Dolan M.J. Laboratory diagnosis of Bartonella infections. Clin Lab Med 2002;22:937-62.

15. Metzkor-Cotter E., Kletter Y., Avidor B., et al. Long-term serological analysis and clinical follow-up of patients with cat-scratch disease. Clin Infect Dis 2003;37:1149-54.

16. Rolain J.M., Gouriet F., Enea M., et al. Detection by immunofluorescence assay of Bartonella henselae in lymph nodes from patients with cat scratch disease. Clin Diagn Lab Immunol 2003; 10:686-91.

17. Gielen J., Wang X.L., Vanhoenacker F., et al. Lymphadenopathy at the medial epitrochlear region in cat-scratch disease. Eur Radiol 2003; 13:1363-9.

18. Bass J.W., Freitas B.C., Freitas A.D., et al. Prospective randomized double blind placebo-controlled evaluation of azithromycin for treatment of cat-scratch disease. Pediatr Infect Dis J 1998; 17:447-52.

19. Maurin M., Gasquet S., Ducco C., Raoult D. Minimal inhibitory concentrations of 28 antibiotic compounds to 14 Bartonella (formerly Rochalimaea) isolates. Antimicrob Agents Chemother 1995; 39:2387-91.

20. Lerdluedeeporn P., Krogstad P., Roberts R.L., Stiehm E.R. Oral corticosteroids in cat-scratch disease. Clin Pediatr (Phila) 2003;42:71-3.

21. Gonzalez B.E., Correa A.G., Kaplan S.L. Cat-scratch disease occurring in three siblings simultaneously. Pediatr Infect Dis J 2003;22:467-8. 